\title{
In vitro assessment of the antimicrobial activity of wound dressings: influence of the test method selected and impact of the pH
}

\author{
Cornelia Wiegand • Martin Abel · Peter Ruth • \\ Peter Elsner · Uta-Christina Hipler
}

Received: 19 March 2014/Accepted: 3 August 2014/Published online: 13 January 2015

(C) The Author(s) 2014. This article is published with open access at Springerlink.com

\begin{abstract}
Antibacterial activity of dressings containing antimicrobials is mostly evaluated using in vitro tests. However, the various methods available differ significantly in their properties and results obtained are influenced by the method selected, micro-organisms used, and extraction method, the degree of solubility or the diffusability of the test-compounds. Here, results on antimicrobial activity of silver-containing dressings obtained by agar diffusion test (ADT), challenge tests (JIS L 1902, AATCC 100), and extraction-based methods (microplate laser nephelometry (MLN), luminescent quantification of bacterial ATP (LQbATP)) using Staphylococcus aureus and Pseudomonas aeruginosa were evaluated. Furthermore, the effect of the $\mathrm{pH}$ on antibacterial efficacy of these dressings was investigated. All silver-containing dressings exerted antimicrobial activity in all in vitro tests and results correlated considerably well. Differences were observed testing the agent-free basic materials. They did not exhibit any antimicrobial effects in the ADT, MLN or LQbATP, since these methods depend on diffusion/extraction of an active agent. However, they showed a strong antimicrobial effect in the challenge tests as they possess a high absorptive capacity, and are able to bind and sequester micro-organisms present. Therefore, it seems recommendable to choose several tests to distinguish whether a material conveys an active effect or a passive mechanism. In addition, it could be shown that release of silver and its antimicrobial
\end{abstract}

C. Wiegand $(\bowtie) \cdot$ P. Elsner $\cdot$ U.-C. Hipler

Department of Dermatology, University Hospital Center Jena,

Erfurter Str. 35, 07740 Jena, Germany

e-mail: C.Wiegand@med.uni-jena.de

M. Abel · P. Ruth

Lohmann \& Rauscher GmbH \& Co. KG, Westerwaldstraße 4, 56579 Rengsdorf, Germany efficacy is partially pH-dependent, and that dressings themselves affect the $\mathrm{pH}$. It can further be speculated that dressings' effects on $\mathrm{pH}$ and release of silver ions act synergistically for antimicrobial efficacy.

$\begin{array}{ll}\text { Abbreviations } \\ \text { AATCC } & \begin{array}{l}\text { American Association of Textile Chemists and } \\ \text { Colorists }\end{array} \\ \text { ADT } & \text { Agar diffusion test } \\ \text { CMC } & \text { Carboxymethylcellulose } \\ \text { JIS } & \text { Japanese Industrial Standard } \\ \text { LQbATP } & \text { Luminometric quantification of bacterial ATP } \\ \text { MLN } & \text { Microplate laser nephelometry } \\ \text { TLC } & \text { Lipidocolloid technology matrix } \\ \text { PU } & \text { Polyurethane } \\ \text { RNU } & \text { Relative nephelometric units } \\ \text { ZOI } & \text { Zone of inhibition }\end{array}$

\section{Introduction}

Treatment of wound infections has an important role in wound management. A high bioburden will interrupt the normal wound healing process and may lead to the formation of persistent, non-healing wounds [1-3]. Hence, dressings containing antimicrobial substances, most commonly silver, are increasingly utilized. Silver $\left(\mathrm{Ag}^{+}\right)$is effective against a broad range of micro-organisms such as yeast, mold and bacteria, including MRSA and VRE, when it is provided in appropriate concentration $[1,4,5]$.

The antibacterial activity of these dressings is mostly evaluated using in vitro tests such as the agar diffusion test (ADT), challenge tests like the JIS L 1902 or the AATCC 
100, as well as new methods such as microplate laser nephelometry (MLN) and luminometric quantification of bacterial ATP (LQbATP). These tests allow a direct comparison of the effects of the dressings on the microorganisms. Ideally, they are simple, rapid, reproducible, inexpensive, and enable handling of a range of sample quantities [6]. However, the various test methods available differ significantly in their properties and hence in their outcome. The results obtained are influenced by the method selected and the microorganisms used as well as by the extraction method or the degree of solubility or diffusability of each test-compound [6].

The agar diffusion test (ADT) can be utilized to measure the effect of an antimicrobial sample against microorganisms grown on culture plates [7, 8]. Therefore, microorganisms are swabbed uniformly across a culture plate and the test sample is applied onto the agar. The antimicrobial agent then has to diffuse from its point of application into the agar. The concentration of the agent will be highest next to the application point, and will decrease with increasing distance. If the agent is effective against the microorganisms at a certain concentration, no colonies will grow where the concentration in the agar is greater than or equal to the effective concentration. This is the zone of inhibition (ZOI). Accordingly, the size of the ZOI can be used to rate the agent's antimicrobial efficacy: the larger the ZOI, the more effective the agent. This method further strictly depends on the diffusion of the antimicrobial agent in the wound dressing. Large or highly charged molecules, e.g. PHMB, might exhibit lower diffusion capacities compared to small ions such as $\mathrm{Ag}^{+}$. Moreover, permanently bound agents cannot diffuse from the sample into the agar and might not elicit a result in the ADT while showing a high antimicrobial activity in challenge tests.

Challenge tests, such as the JIS L 1902 or AATCC 100, analyse the antimicrobial efficacy of the material after direct contact with the microorganisms over a respective time period. In contrast to the ADT, they are independent from the diffusion properties of the antimicrobial agent. In addition, they allow a quantitative evaluation of antimicrobial activity as results are retrieved as percentage reduction of microbial counts [9] or inhibition of microbial growth in log-scale [10]. However, these test methods are labour-intensive and time-consuming resulting in difficulties if large sample numbers have to be processed.

High-throughput screening approaches for determining the reaction of microorganisms to antimicrobial agents mostly relay on the measurement of the cellular adenosine triphosphate (ATP) content or observation of microbial growth by determination of turbidity. As ATP is found in all living and metabolic active cells, it can be used to determine the amount of viable microbial cells present. It can be quantified using a bioluminescence assay comprised of the enzyme luciferase from Photinus pyralis and Dluciferin, the enzyme's substrate. Luciferin is converted into oxyluciferin in an ATP-, $\mathrm{Mg}^{2+}$ - and oxygen dependent reaction which generates a yellow-green luminescent light signal [11, 12]. The amount of emitted light is directly proportional to the ATP content [11, 13], and hence, a linear function of the number of living cells in the suspension [14].

However, the turbidity of the respective medium itself can be used to monitor the growth of microorganisms. While turbidimetry obeys Beer's Law and requires relatively high concentrations of particles to be measured, nephelometry is a direct method to measure light scattered already by relatively low amounts of particles in solution at right or forward angle to a laser beam. The most common application of laser-based nephelometry in microplate format, designated as microplate laser nephelometry (MLN) is the fully automated solubility screen in HTS laboratories [15]. Nephelometry is further used in clinical chemistry to determine serum immunoglobulin (IgA, IgG, IgM), complement components (C3, C4), acute phase reactant proteins (CRP, transferring), albumin, and $\alpha-1$ antitrypsin by protein precipitation or in organic chemistry to quantify macromolecules, e.g. monitoring of a polymerisation reaction. MLN presents a valuable tool to investigate the effect of antimicrobial substances on the growth of microorganisms, as it allows high-throughput screening, incubation over a prolonged time period, and in situ-monitoring of changes in the dose-response curves as well as the $\mathrm{IC}_{50}$, the half maximum inhibitory concentrations [16-19]. Both, MLN and LQbATP are performed in solution and wound dressing extracts were prepared according to DIN EN ISO 10993-12 as previously reported [20, 21].

The presented study describes the comparison of the results of antimicrobial activity of silver-containing dressings obtained by ADT, JIS L 1902 and AATCC 100, as well as MLN and LQbATP using Staphylococcus aureus and Pseudomonas aeruginosa, which are the most prominent bacteria in wound infection [22, 23], as model organisms.

Moreover, as only limited knowledge exists concerning the effect of the wound $\mathrm{pH}$ on the antibacterial efficacy of antimicrobial-containing dressings, ADT and MLN were elected to investigate the influence of $\mathrm{pH}$ on antimicrobial activity of silver-containing dressings. Chronic wounds most commonly have a $\mathrm{pH}$ range of 6.5-8.5 [24, 25]. This shift towards higher $\mathrm{pH}$ values in chronic wounds compared to acute wounds is called 'alkaline shift' and is thought to be due to both, tissue necrosis and the presence of microorganisms. Recent studies relating to the influence of the $\mathrm{pH}$ on the performance of antiseptics revealed that 
bactericidal activity of chlorhexidine and octenidine was mainly $\mathrm{pH}$-independent in $\mathrm{pH}$ range from 5.0 to 9.0 while a most pronounced influence was observed for polihexanide and PVP-iodine (own unpublished results). In addition, in vitro studies have shown that wound dressings can have significant effects on the $\mathrm{pH}$ [26]. Hence, it is of interest to further determine the effect of the dressing itself on the surrounding $\mathrm{pH}$ as the establishment of a low physiological $\mathrm{pH}$ might also aid wound healing.

\section{Materials and methods}

\subsection{Materials}

The following wound dressings were selected for this study: alginate (Suprasorb ${ }^{\circledR}$ A, Lohmann \& Rauscher GmbH \& Co. $\mathrm{KG}$, Germany) and alginate + ionic-Ag (Suprasorb $^{\circledR}$ A + Ag, Lohmann \& Rauscher GmbH \& Co. KG, Germany), alginate + nano-Ag (Acticoat ${ }^{\diamond}$ Absorbent, Smith \& Nephew GmbH, Germany), sodium carboxymethylcellulose (CMC) (AQUACEL ${ }^{\circledR}$, ConvaTec GmbH, Germany) CMC with $\mathrm{Ag}^{+}$(AQUACEL ${ }^{\circledR} \mathrm{Ag}$, ConvaTec GmbH, Germany) as well as polyurethane (PU)-foam with TLC $\left(\mathrm{UrgoCell}^{\circledR}\right.$, URGO GmbH, Germany) and PU-foam with $\mathrm{TLC} / \mathrm{Ag}^{+}$ (UrgoCell $^{\circledR}$ silver, URGO GmbH, Germany). Active ingredients and wound dressing basic materials are specified in Table 2 according to the manufacturers' descriptions.

Staphylococcus aureus ATCC 6538 and P. aeruginosa ATCC 27853 were obtained from the DSMZ (Deutsche Sammlung von Mikroorganismen und Zellkulturen, Germany). For cultivation of bacteria, special peptone and "lab-lemco" powder for preparation of caso-bouillon and bacteriological agar were purchased from Oxoid (UK). Columbia agar plates with $5 \%$ sheep blood and $\mathrm{MH} 2$ agar plates were acquired from Biomeriéux (France).

$0.9 \% \mathrm{NaCl}$ solution was purchased from Fresenius Kabi Deutschland $\mathrm{GmbH}$ (Germany). $1 \mathrm{~N} \mathrm{HCl}, 1 \mathrm{~N} \mathrm{NaOH}$, and Tween 20 were obtained from Carl Roth GmbH (Germany).

\subsection{Culture of $S$. aureus and P. aeruginosa}

Seed stocks of $S$. aureus and $P$. aeruginosa were kept on Columbia agar plates. For experiments, overnight cultures were prepared by inoculation of $20 \mathrm{~mL}$ caso-bouillon ( $\mathrm{pH} 7.0$ ) with 1-2 colonies of the respective test organism. Overnight cultures were incubated for $16 \mathrm{~h}$ at $37^{\circ} \mathrm{C}$ under shaking.

\subsection{Evaluation of antibacterial activity by ADT}

The agar diffusion test (ADT) was performed in accordance with the DIN 58940-3. Staphylococcus aureus and $P$. aeruginosa overnight cultures were diluted 1:100 to adjust the working suspensions to a microbial count of app. $1-5 \times 10^{6} \mathrm{cfu} / \mathrm{mL} .100 \mu \mathrm{L}$ of these suspensions were plated on MH2-agar plates and left to dry for $10 \mathrm{~min}$. Afterwards aseptically prepared dressing samples (with a diameter of $6 \mathrm{~mm}$ ) were placed onto the inoculated agar plates along with the negative control (additive-free bio-discs; Biomeriéux, France) and the respective positive control ( $S$. aureus: bio-discs with $30 \mu \mathrm{g}$ vancomycin, $P$. aeruginosa: bio-discs with $10 \mu \mathrm{g}$ gentamycin; Biomeriéux, France). All samples and controls were wetted with $20 \mu \mathrm{L}$ of $0.9 \% \mathrm{NaCl}$. The plates were incubated for $24 \mathrm{~h}$ at $37^{\circ} \mathrm{C}$. Afterwards the zone of inhibition (ZOI) was measured in $\mathrm{mm}$ and all plates were photographed for documentation.

\subsection{Assessment of the antibacterial activity according} to JIS L 1902

Testing for antibacterial activity was carried out in accordance to the Japanese industrial standard (JIS L 1902:2002, "Testing method for antibacterial activity of textiles") as reported previously [20]. In brief, overnight cultures of $S$. aureus and $P$. aeruginosa were diluted 1:1,000 to adjust a microbial count of app. $1-2 \times 10^{5} \mathrm{cfu} / \mathrm{mL}$. For experiments, $400 \mathrm{mg}$ samples of the wound dressings were aseptically prepared and inoculated with $200 \mu \mathrm{L}$ test microbe solution and incubated for $24 \mathrm{~h}$ at $37{ }^{\circ} \mathrm{C}$ under aerobic conditions. Polyester material (I.T.S. Textilhandels $\mathrm{GmbH}$, Austria) was used as growth control. For determination of the germ number, the incubated samples were extracted in $0.9 \% \mathrm{NaCl}$ solution supplemented with $0.2 \%$ Tween 20. Serial dilutions were plated onto Columbia agar plates and incubated for $24 \mathrm{~h}$ at $37^{\circ} \mathrm{C}$. Subsequently, colonies were counted, total cfu (colony forming units) determined, and growth reduction calculated according to Eq. (1). A logarithmic microbial growth reduction of less than 0.5 represents no antibacterial activity. Values between 0.5 and 1 are rated as a slight, values greater than 1 and less or equal to 3 as a significant, and a log reduction greater than 3 as a strong antibacterial activity.

$\log$ growth reduction ${ }_{(24 \mathrm{~h})}=\log \mathrm{cfu}(\text { negative control })_{(24 \mathrm{~h})}$

$$
-\log \text { cfu(sample) }{ }_{(24 h)}
$$

2.5 Measurement of the antibacterial efficacy according to AATCC 100

Antibacterial efficacy was determined according to the AATCC test method 100 (AATCC 100-2004, "Assessment of antibacterial finishes on textile materials"). Staphylococcus aureus and $P$. aeruginosa overnight cultures were diluted 1:1,000 to adjust a microbial count of app. 1$2 \times 10^{5} \mathrm{cfu} / \mathrm{mL}$. For experiments, circular swatches 
( $d=5 \mathrm{~cm})$ of the wound dressings were aseptically prepared and incubated with $1 \mathrm{~mL}$ test microbe solution for $24 \mathrm{~h}$ at $37{ }^{\circ} \mathrm{C}$ under aerobic conditions. Polyester material (I.T.S. Textilhandels GmbH, Austria) was used as growth control. After incububation, samples are transferred to a neutralizing solution $(0.9 \% \mathrm{NaCl}$ solution with $0.2 \%$ Tween 20). For determination of the germ number, serial dilutions were plated onto Columbia agar plates and incubated for $24 \mathrm{~h}$ at $37{ }^{\circ} \mathrm{C}$. Subsequently, colonies were counted, total cfu (colony forming units) determined, and growth reduction calculated according to Eq. (2). A germ reduction of less than $50 \%$ represents no antibacterial efficacy. Values between 50 and $90 \%$ are rated as a significant and a germ reduction greater than $90 \%$ as a strong antibacterial efficacy. Furthermore, to allow comparison between AATCC 100 and JIS L 1902, the results retrieved were also subjected to calculation of antibacterial activity according to Eq. (1).

Germ reduction $[\%]=\frac{\left(\mathrm{cfu}(\text { control })_{(0 \mathrm{~h})}-\mathrm{cfu}\left(\text { sample }_{(24 \mathrm{~h})}\right)\right.}{\operatorname{cfu}(\operatorname{control})_{(0 \mathrm{~h})}}$ $\times 100$

2.6 Determination of the antibacterial effect of wound dressings by MLN and LQbATP

MLN and LQbATP are solution-based methods, hence, wound dressing extracts were prepared according to the standard used for evaluation of textile cytotoxicity (DIN EN ISO 10993-12) as previously reported [20, 21]. Briefly, $1 \mathrm{~g}$ of each aseptically treated wound dressing was incubated in $50 \mathrm{~mL}$ caso-bouillon in Erlenmeyer flasks (Greiner, Germany) at $37^{\circ} \mathrm{C}$ for $24 \mathrm{~h}$ under shaking (ThermoBath, GFL, Germany). Afterwards, each wound dressing extract was filtered over gauze by centrifugation at $1,000 \mathrm{rpm}$ to remove any insoluble material residues. This filtrate was then sterilized by passage through a $0.2 \mu \mathrm{m}$ filter and distinguished as original extract (100\%).

Microplate laser nephelometry (MLN) was performed in accordance with NCCLS M27-A2 and DIN EN 27027 as reported previously [14, 16, 17]. In brief, $100 \mu \mathrm{L}$ of each extract were put in triplicate into the respective wells of a sterile, clear 96-well microplate (Greiner bio-one, Germany). Blanks for each extract tested were run at every assay. $100 \mu \mathrm{L}$ of $S$. aureus or $P$. aeruginosa cell suspension (overnight cultures diluted to a microbial count of app. $5 \times 10^{3} \mathrm{cfu} / \mathrm{mL}$ ) were put in the respective wells of the 96-well microplate containing the wound dressing extracts. Microplates were covered with a clear adhesive film (Greiner bio-one, Germany). The adhesive film was punctured with a 25-gauge needle at the right brim of the well to allow gas exchange. Microplates were then placed in the microplate lasernephelometer (NEPHELOstar
Galaxy, BMG LABTECH, Germany) and incubated for $24 \mathrm{~h}$ at $37{ }^{\circ} \mathrm{C}$. During incubation, microplates were shaken in the instrument except for the duration of the hourly measurement.

$$
\begin{aligned}
& \text { Microbial growth }(\mathrm{MLN})[\%] \\
& =\frac{\sum[\mathrm{RNU}]_{1-24 \mathrm{~h}}(\text { sample })}{\sum[\mathrm{RNU}]_{1-24 \mathrm{~h}}(\text { control })} \times 100
\end{aligned}
$$

The effect of the wound dressing extracts on microbial viability was further determined by luminometric quantification of the bacterial ATP content (LQbATP) [14, 16] using the BacTiter-Glo ${ }^{\mathrm{TM}}$ Assay (Promega, Mannheim, Germany), which is based on the detection of light generated by the ATP dependent enzymatic conversion of D-luciferin to oxyluciferin by firefly luciferase. After MLN measurements, microplates were equilibrated at room temperature under agitation. $10 \mu \mathrm{L}$ of the contents of each well were transferred into a white 96-well microplate (NUNC MaxiSorp $^{\mathrm{TM}}$; Thermo Fisher Scientific, Germany) and mixed with $90 \mu \mathrm{L}$ caso-bouillon. Afterwards, $100 \mu \mathrm{L}$ BacTiterGlo $^{\text {TM }}$ Reagent was added to each well. The microplates were further incubated under agitation for $5 \mathrm{~min}$. Thereafter, luminescence was recorded using the LUMIstar Galaxy (BMG LABTECH, Germany). The ATP concentration per well was calculated using an ATP standard curve.

Microbial growth (LQbATP) $[\%]$
$=\frac{\operatorname{ATP}[n M]_{24 \mathrm{~h}}(\text { sample })}{\operatorname{ATP}[n M]_{24 \mathrm{~h}}(\text { control })} \times 100$

2.7 Evaluation of the $\mathrm{pH}$ effect on the antibacterial activity of silver dressings' efficacy using ADT and MLN

The agar diffusion test (ADT) was carried out as described in Evaluation of antibacterial activity by $A D T$ with the difference that instead of $\mathrm{MH} 2$ agar plates, caso agar plates with specific $\mathrm{pH}$ were employed. Caso-bouillon $(\mathrm{pH} 7.0)$ with different $\mathrm{pH}$ was prepared by addition of $\mathrm{HCl}$ yielding caso-bouillon with pH 6.0 and 5.0, and by adding $\mathrm{NaOH}$ producing $\mathrm{pH} 8.0$ and 9.0, respectively. These media were supplemented with $1.5 \%$ agar, carefully heated until the agar was dissolved, and then cast into Petri dishes (Greiner bio-one, Germany), $10 \mathrm{~mL}$ per dish, under sterile conditions. The prepared caso-agar plates were kept at $4{ }^{\circ} \mathrm{C}$ until use.

Microplate laser nephelometry (MLN) was executed according to the section Determination of the antibacterial effect of wound dressings by MLN and LQbATP using casobouillon with different $\mathrm{pH}$. Caso-bouillon ( $\mathrm{pH}$ 7.0) with specific $\mathrm{pH}$ was prepared by addition of $\mathrm{HCl}$ yielding casobouillon with $\mathrm{pH} 6.0$, and by adding $\mathrm{NaOH}$ producing $\mathrm{pH} 8.0$ and 9.0, respectively. These media were used in the extraction step as well as for preparation of the $S$. aureus or $P$. aeruginosa 


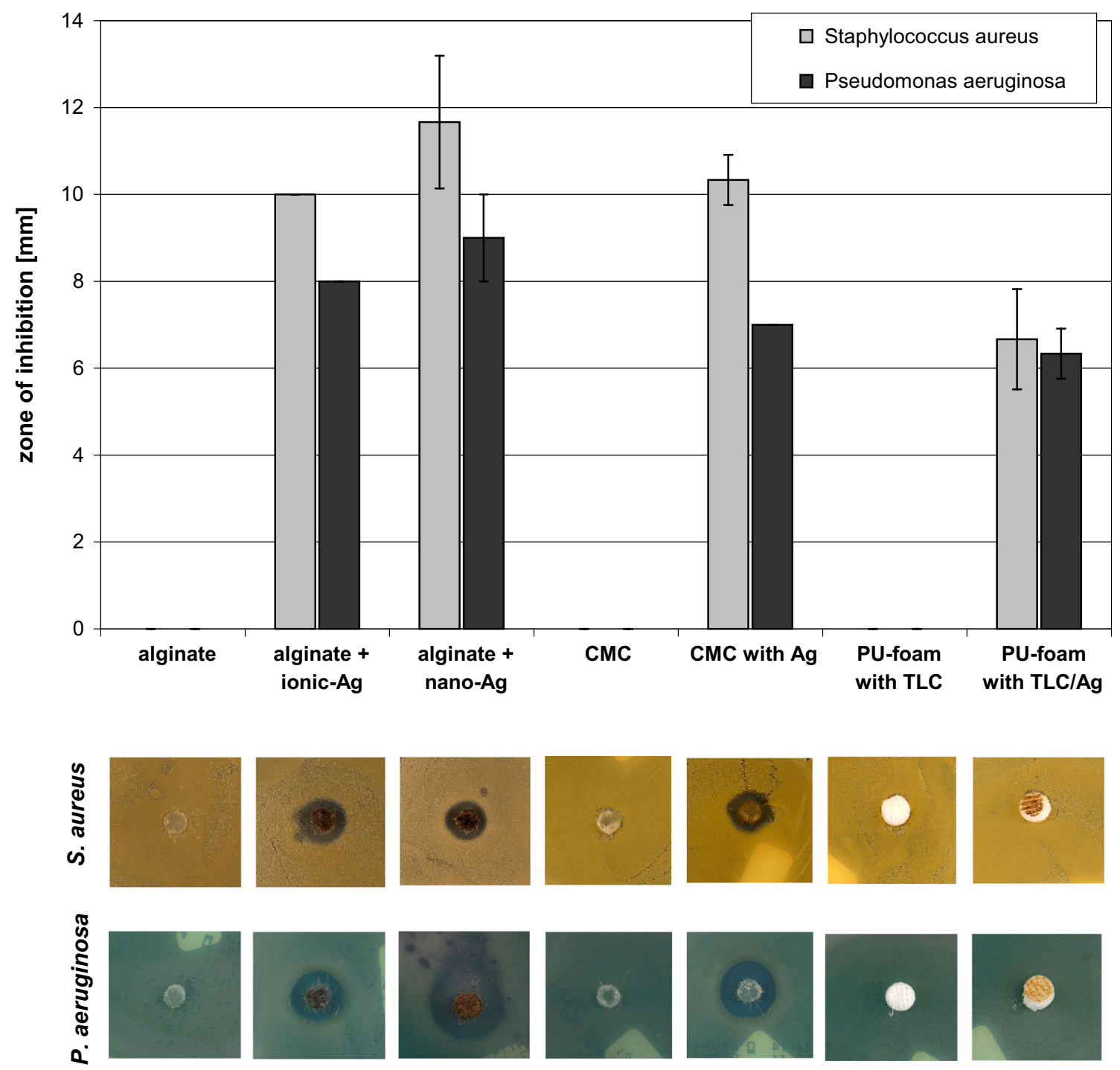

Fig. 1 For the ADT, MH2 agar plates were inoculated with $S$. aureus or $P$. aeruginosa and incubated with the wound dressing samples at $37^{\circ} \mathrm{C}$ for $24 \mathrm{~h}$. Afterwards, the zone of inhibition (ZOI) was measured. Pictures show the photographic documentation of representative ADT results

cell suspensions with a microbial count of app. $5 \times 10^{3} \mathrm{cfu} /$ $\mathrm{mL}$. The half maximal inhibitory concentration $\left(\mathrm{IC}_{50}\right)$ of the dressing extracts under the experimental conditions used was calculated from the growth curves over $24 \mathrm{~h}$. The 'area under the curve' was determined from the results for each antiseptic concentration tested and calculated as percentage of the untreated control. This was used to realize a dose-response curve for each antiseptic tested, from which the $\mathrm{IC}_{50}$ was calculated using a logistic fit function $(\mathrm{y}=\mathrm{A} 2+(\mathrm{A} 1-\mathrm{A} 2) /$ $\left(1+(\mathrm{x} / \mathrm{x} 0)^{\wedge} \mathrm{p}\right)$; $\mathrm{A} 1$ : upper limit, $\mathrm{A} 2$ : lower limit, $\mathrm{x} 0$ : $\mathrm{IC}_{50}$, $\mathrm{p}$ : slope of the curve; Origin 7.5, OriginLab, U.S.).

\subsection{Statistical analysis}

One-way analysis of variance was carried out to determine statistical significances (Microsoft ${ }^{\circledR}$ Excel 2000). Differences were considered statistically significant at a level of $P<0.05$.
Asterisks indicate significant deviations from the control at the respective incubation time $(* P<0.05 ; * * P<0.01$; $* * * P<0.001)$.

\section{Results}

3.1 Silver containing dressings exhibit a comparable antibacterial activity in the ADT

Dressings without active agent, such as alginate, CMC and PU-foam with TLC, had no effect in the ADT (Fig. 1). On the other hand, the silver-containing dressings, alginate + ionic- $\mathrm{Ag}$, alginate + nano- $\mathrm{Ag}$ and CMC with $\mathrm{Ag}^{+}$, exhibited a formation of a distinct zone of inhibition (ZOI) for both, S. aureus and P. aeruginosa. It was further noted 
Table 1 Testing of antibacterial activity according to JIS L 1902 as log-reduction of microbial growth and according to AATCC 100 as decrease of viable germs in [\%] revealed a strong antimicrobial effect for alginate, alginate + ionic- $\mathrm{Ag}$, and alginate + nano- $\mathrm{Ag}$ against both $S$. aureus and $P$. aeruginosa as well as for $\mathrm{CMC}$ and $\mathrm{CMC}$ with $\mathrm{Ag}^{+}$

\begin{tabular}{|c|c|c|c|c|c|c|}
\hline & \multicolumn{2}{|l|}{ JIS L 1902} & \multicolumn{4}{|l|}{ AATCC 100} \\
\hline & \multicolumn{2}{|c|}{$\begin{array}{l}\text { Microbial growth reduction }[\log \\
\text { cfu] }\end{array}$} & \multicolumn{2}{|c|}{ Germ reduction [\%] } & \multicolumn{2}{|c|}{$\begin{array}{l}\text { Microbial growth reduction [log cfu] (according to } \\
\text { JIS L 1902) }\end{array}$} \\
\hline & S. aureus & P.aeruginosa & S. aureus & P.aeruginosa & S. aureus & P.aeruginosa \\
\hline Control & $0 \pm 0.2$ & $0 \pm 0.2$ & $0 \pm 1.0$ & $0 \pm 1.0$ & $0 \pm 0.2$ & $0 \pm 0.2$ \\
\hline Alginate & $7.0 \pm 0$ & $7.0 \pm 0$ & $100 \pm 0$ & $100 \pm 0$ & $6.4 \pm 0$ & $8.9 \pm 0$ \\
\hline Alginate + ionic-Ag & $7.0 \pm 0$ & $7.0 \pm 0$ & $100 \pm 0$ & $100 \pm 0$ & $6.4 \pm 0$ & $8.9 \pm 0$ \\
\hline Alginate + nano-Ag & $7.0 \pm 0$ & $7.0 \pm 0$ & $100 \pm 0$ & $100 \pm 0$ & $6.4 \pm 0$ & $8.9 \pm 0$ \\
\hline $\mathrm{CMC}$ & $4.0 \pm 0.2$ & $4.0 \pm 0.2$ & $100 \pm 0$ & $100 \pm 0$ & $6.4 \pm 0$ & $8.9 \pm 0$ \\
\hline $\mathrm{CMC}$ with $\mathrm{Ag}^{+}$ & $4.0 \pm 0.7$ & $4.0 \pm 0.7$ & $100 \pm 0$ & $100 \pm 0$ & $6.4 \pm 0$ & $8.9 \pm 0$ \\
\hline PU-foam with TLC & $0.7 \pm 0.2$ & $0.7 \pm 0.2$ & $99.8 \pm 0.1$ & $0 \pm 0.9$ & $4.8 \pm 1.7$ & $2.2 \pm 0.3$ \\
\hline PU-foam with $\mathrm{TLC} / \mathrm{Ag}^{+}$ & $3.3 \pm 0.5$ & $3.3 \pm 0.5$ & $100 \pm 0$ & $100 \pm 0$ & $6.4 \pm 0$ & $8.9 \pm 0$ \\
\hline
\end{tabular}

PU-foam with TLC already had a slight antibacterial effect that was significantly increased in the silver-containing product PU-foam with TLC/ $\mathrm{Ag}^{+}$. For comparison, the results of the AATCC 100 have also been evaluated according to the JIS L 1902 to yield log-reduction of microbial growth

that the effect on $S$. aureus was slightly higher compared to $P$. aeruginosa. In contrast, for PU-foam with $\mathrm{TLC} / \mathrm{Ag}^{+}$ inhibition of bacterial growth was only found directly under the sample and no ZOI was formed.

\subsection{Reduction of microbial growth in the challenge tests JIS L 1902 and AATCC 100}

A high effectiveness of both, agent-free and silver-containing dressings was observed in the challenge tests (Table 1). According to JIS L 1902, alginate, alginate + ionic-Ag, alginate + nano- $\mathrm{Ag}, \mathrm{CMC}, \mathrm{CMC}$ with $\mathrm{Ag}^{+}$and PU-foam with $\mathrm{TLC} \mathrm{Ag}^{+}$possess a strong antibacterial activity against $S$. aureus. Furthermore, a slight effect of PU-foam with TLC could also be observed. All dressings demonstrated a distinctly higher effect on $P$. aeruginosa compared to $S$. aureus and achieved a higher log reduction of the gram-negative bacteria compared to the gram-positive bacteria. Similar results were obtained in the AATCC 100 test (Table 2), All dressings showed a strong antibacterial efficacy against both, $S$. aureus and $P$. aeruginosa, with a growth reduction larger $90 \%$.

For comparison of the results from the JIS L 1902 and the AATCC 100, the data from the latter was evaluated according to the log-reduction suggested by the JIS L 1902 (Table 1). The AATCC 100 differs from the JIS L 1902 in the way that more test material is used. Hence, mostly a higher effectiveness of the dressings was found in the AATCC 100 compared to the JIS L 1902 in the case of $S$. aureus as well as for $P$. aeruginosa.

\subsection{Determination of antibacterial activity using MLN and LQbATP}

Microplate laser nephelometry (MLN) was used to monitor the growth of S. aureus (Fig. 2a) and P. aeruginosa (Fig. 2b) under the influence of the dressing extracts. In accordance to the ADT, a bactericidal effect was only observed in the case of the silver-containing dressings. The antibacterial activity of the extracts further depended on the extractability of the silver in the dressings. A complete inhibition of $S$. aureus and $P$. aeruginosa growth was achieved by extracts of alginate + ionic-Ag and alginate + nano-Ag. The extract of CMC with $\mathrm{Ag}^{+}$demonstrated a significant reduction of $S$. aureus growth; however, silver concentrations reached were not high enough to abolish $P$. aeruginosa progeny. In contrast, extract of PU-foam with $\mathrm{TLC}_{\mathrm{Ag}}{ }^{+}$accomplished a higher effect against $P$. aeruginosa compared to $S$. aureus.

Using LQbATP, similar results were obtained in regard to the basic materials that did not significantly affect bacterial growth (Fig. 3). Moreover, extracts of alginate + ionic-Ag and alginate + nano-Ag also demonstrated a complete reduction of $S$. aureus and $P$. auruginosa. However, differing results were found for the extracts of CMC with $\mathrm{Ag}^{+}$and PU-foam with $\mathrm{TLC} / \mathrm{Ag}^{+}$ against both, S. aureus (Fig. 3a) and $P$. aeruginosa (Fig. 3b). Here, the LQbATP did not show an equal reduction of microbial growth compared to the MLN where the inhibition of bacterial growth was clearly observable. 
Table 2 Wound dressing extracts were prepared in casobouillon with $\mathrm{pH}$ 6.0, 7.0, 8.0, or 9.0 by incubation for $24 \mathrm{~h}$ at $37{ }^{\circ} \mathrm{C}$

Afterwards, the actual $\mathrm{pH}$ was determined

\begin{tabular}{|c|c|c|c|c|c|c|}
\hline \multirow[t]{2}{*}{ Wound dressing } & \multirow[t]{2}{*}{ Basic material } & \multirow[t]{2}{*}{$\begin{array}{l}\text { Active } \\
\text { ingredient }\end{array}$} & \multicolumn{4}{|c|}{$\begin{array}{l}\text { Effect on actual } \mathrm{pH} \\
\text { value at } \mathrm{pH}\end{array}$} \\
\hline & & & 6.0 & 7.0 & 8.0 & 9.0 \\
\hline Alginate + ionic- $\mathrm{Ag}$ & Alginate & Ionic silver & 5.7 & 6.5 & 7.2 & 7.7 \\
\hline Alginate + nano-Ag & Alginate & $\begin{array}{l}\text { Nano-crystalline } \\
\text { silver }\end{array}$ & 7.6 & 7.9 & 8.2 & 8.4 \\
\hline $\mathrm{CMC}$ with $\mathrm{Ag}^{+}$ & $\begin{array}{l}\text { Sodium carboxymethyl } \\
\text { cellulose (CMC) }\end{array}$ & $\mathrm{Ag}^{+}$ & 5.6 & 6.1 & 7.1 & 8.1 \\
\hline PU-foam with $\mathrm{TLC} / \mathrm{Ag}^{+}$ & $\begin{array}{l}\text { Polyurethane (PU) foam } \\
\text { with TLC (lipidocolloid matrix) }\end{array}$ & $\mathrm{Ag}^{+}$ & 6.2 & 7.2 & 7.8 & 8.6 \\
\hline
\end{tabular}

\subsection{Employing ADT and MLN to evaluate the $\mathrm{pH}$} effect on antibacterial activity of silver-dressings

For testing the effect of the $\mathrm{pH}$ on antibacterial efficacy in the ADT, caso-agar plates with $\mathrm{pH}$ 5.0, 6.0, 7.0, 8.0, and 9.0 were inoculated with S. aureus (Fig. 4a) or P. aeruginosa (Fig. 4b). Alginate + nano-Ag caused an average ZOI of $10 \mathrm{~mm}$ and was equally effective against $S$. aureus and $P$. aeruginosa. No change in the antibacterial effect was further observed for PU-foam with $\mathrm{TLC}_{\mathrm{Agg}}{ }^{+}$at the different $\mathrm{pH}$. In contrast, $\mathrm{CMC}$ with $\mathrm{Ag}^{+}$exhibited an increased ZOI against $S$. aureus at $\mathrm{pH} 9.0$ and against $P$. aeruginosa at both, $\mathrm{pH} 5.0$ and $\mathrm{pH}$ 9.0. Interestingly, alginate + ionic-Ag did not reveal a $\mathrm{pH}$-dependency of the ZOI formation in the test against $S$. aureus. However, against $P$. aeruginosa a significant increase of the antibacterial effect from $\mathrm{pH} 5.0$ to $\mathrm{pH} 9.0$ was observed for alginate + ionic-Ag.

It was found that low $\mathrm{pH}$ (5.0) already effectively inhibited microbial growth in solution. While no significant difference in the growth of $S$. aureus and $P$. aeruginosa was observed at $\mathrm{pH}$ 6.0-9.0, their progeny at $\mathrm{pH} 5.0$ was found to be reduced to less than $10 \%$ of the control at $\mathrm{pH} 7.0$ (own unpublished results). Hence, MLN tests were only performed at a $\mathrm{pH}$ range of 6.0-9.0. It could be shown that the silver-dressing extracts possess a pH-dependent antimicrobial activity. For instance, $\mathrm{IC}_{50}$ values of the extracts of PU-foam with $\mathrm{TLC} / \mathrm{Ag}^{+}$significantly decreased from $\mathrm{pH} 6.0$ to $\mathrm{pH} 9.0$ against both, $S$. aureus and $P$. aeruginosa (Fig. 5d). Alginate + ionic-Ag also exhibited an increase in antibacterial activity for $P$. aeruginosa while $\mathrm{IC}_{50}$ values for S. aureus slightly increased from $\mathrm{pH} 6.0$ to $\mathrm{pH} 8.0$ and then again dropped at $\mathrm{pH} 9.0$ (Fig. 5a). In addition, alginate + nano-Ag (Fig. 5b) and CMC with $\mathrm{Ag}^{+}$ (Fig. 5c) showed similar effects against $S$. aureus and $P$. aeruginosa demonstrating a reduction in $\mathrm{IC}_{50}$ values with increasing $\mathrm{pH}$ from 7.0 to 9.0. In both cases, a significantly higher antibacterial activity of the extracts was observed at $\mathrm{pH} 6.0$.

\section{Discussion}

In vitro tests allow the direct comparison of antimicrobial effects of wound dressings. However, the method selected will influence the results obtained and therefore, the evaluation of the antimicrobial activity of a dressing. Here, the antimicrobial activity of silver-containing dressings was compared using standard tests like the agar diffusion test (ADT) and the challenge tests JIS L 1902 and AATCC 100, as well as extraction-based methods such as microplatelaser nephelometry (MLN) and luminometric quantification of bacterial ATP (LQbATP).

In general, all silver-containing dressings exhibited antimicrobial activity in all in vitro tests used. The results correlated considerably well, for instance, a strong antimicrobial effect was determined for alginate + ionic $\mathrm{Ag}$ and alginate + nano-Ag by ADT, JIS L 1902, AATCC 100, MLN as well as LQbATP The PU-foam with TLC/ $\mathrm{Ag}^{+}$inhibited bacterial growth only directly under the sample and no ZOI was formed in the ADT. In accordance, MLN showed that growth of $S$. aureus and $P$. aeruginosa was less affected by the extract of PU-foam with TLC/Ag ${ }^{+}$ compared to the preparations from alginate + ionic-Ag and alginate + nano-Ag. These results indicate that the active agent silver is released in lower amounts by the PUfoam with TLC dressing because it either is present in lesser concentrations or is more closely bound in this material compared to the other dressings with alginate and carboxymethylcellulose. The higher activity of CMC with $\mathrm{Ag}^{+}$against $S$. aureus compared to $P$. aeruginosa in the ADT translated into a stronger effect of the extract on the growth of the gram-positive germ by contrast with the gram-negative test species in the MLN. Minor antibacterial effects of CMC with $\mathrm{Ag}+$ compared to the silver-containing alginates might again be explained by lower amounts of silver ions present as well as effects of the basic materials themselves conveying antibacterial activity. Moreover, delivery of the ionic-Ag in the alginate dressing might be miore readily and the effect of nano-Ag more sustained. Differing results were observed using LQbATP 
Fig. 2 Growth curves of $S$. aureus (a) and $P$. aeruginosa (b) under the influence of the dressing extracts determined by MLN. It was found that extracts of silver-free dressings did not affect the growth of $S$. aureus and $P$. aeruginosa in solution. Extracts of silver-containing alginates effectively diminished bacterial growth, while CMC with $\mathrm{Ag}^{+}$and PU-foam with $\mathrm{TLC} / \mathrm{Ag}^{+}$were only able to inhibit $S$. aureus and $P$. aeruginosa progeny
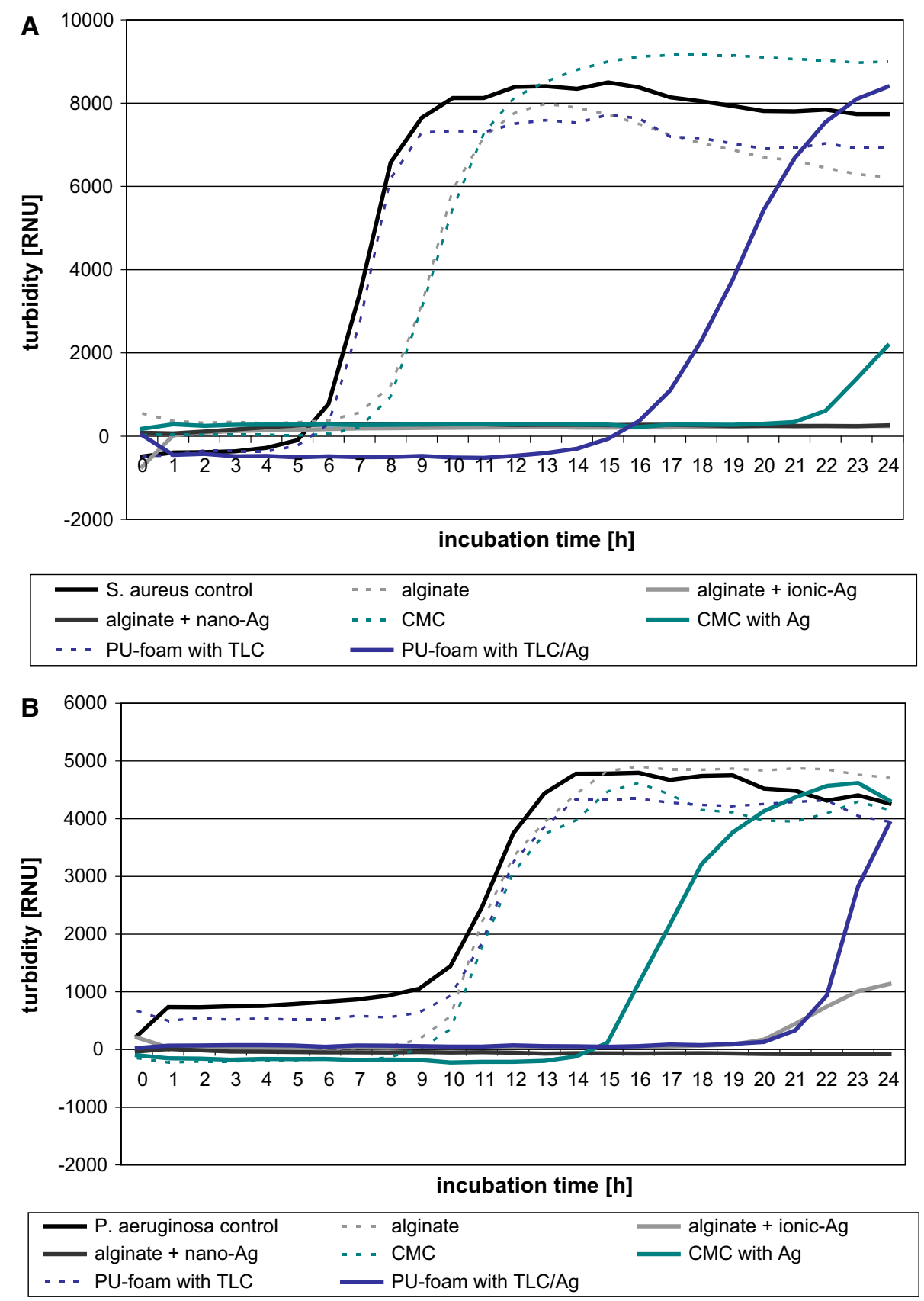

to determine the antimicrobial efficacy of extracts of CMC with $\mathrm{Ag}^{+}$and PU-foam with TLC/Ag ${ }^{+}$compared to MLN. Here, the LQbATP did not show any or only a moderate reduction of microbial growth while MLN clearly indicated the inhibition of bacterial growth. It is most likely that the dressings affect the LQbATP assay by rendering the $\mathrm{pH}$. This could have an influence on the test outcome as the determination of viable bacteria in the assay depends on cell lysis which is as is known $\mathrm{pH}$-sensitive.

As expected, neither the ADT nor the extraction-based methods demonstrated antimicrobial activity of the silver- free dressings. However, antimicrobial effects of the basic materials alginate, carboxymethylcellulose (CMC) and polyurethane with TLC (PU-foam with TLC) were found in the challenge tests. Both, JIS L 1902 and AATCC 100 revealed a strong antimicrobial activity with log-reduction values $>3$ (JIS L 1902) or a reduction of germs $>90 \%$ (AATCC 100) for alginate and CMC against both $S$. aureus and $P$. aeruginosa. The PU-foam with TLC achieved a slight antimicrobial activity against $S$. aureus and a strong effect on $P$. aeruginosa according to JIS L 1902. Solely in the AATCC 100 test, the PU-foam with TLC did not 
Fig. 3 For evaluation of the MLN and LQbATP assay results, data was transformed to read the growth of $S$. aureus (a) and P. aeruginosa (b) in [\%] compared to the medium control. It was found that the basic materials alginate, CMC, and PU-foam with TLC did not significantly affect bacterial growth. Extracts of alginate + ionic- $\mathrm{Ag}$ and alginate + nano-Ag demonstrated a complete reduction of $S$. aureus and $P$. auruginosa. Differing results were found for the extracts of $\mathrm{CMC}$ with $\mathrm{Ag}^{+}$and PU-foam with $\mathrm{TLC} / \mathrm{Ag}^{+}$. Here, the LQbATP did not show an equal reduction of microbial growth compared to MLN where inhibition of bacterial growth was clearly observable
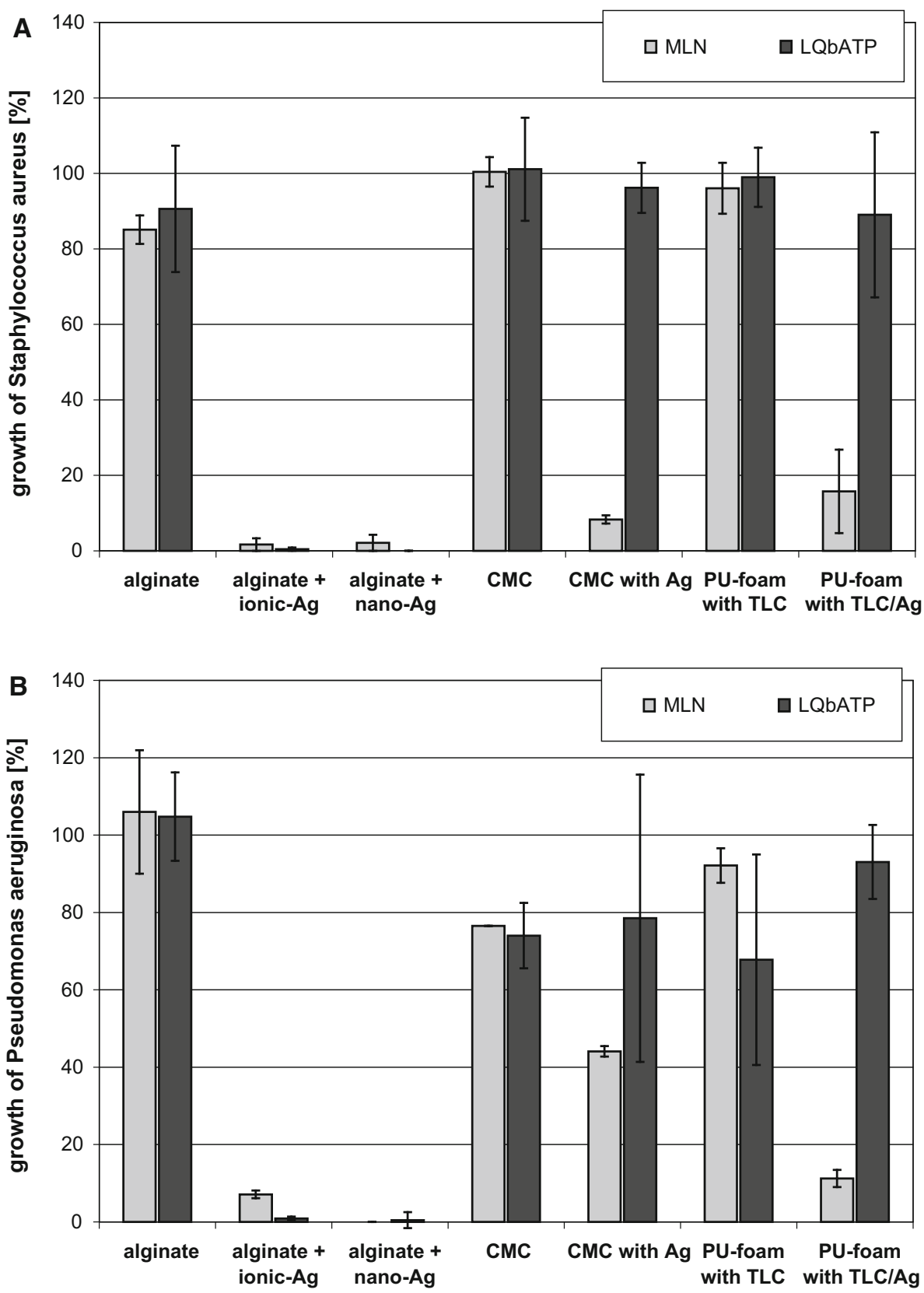

succeed in decreasing the numbers of $P$. aeruginosa. However, at least a significant antimicrobial activity was obtained by evaluation of the same results according to JIS L 1902. This is due to the fact that JIS L 1902 rates the total antibacterial activity where the reduction of micro-organism growth by the test sample is evaluated in comparison to a polyester growth control after the respective incubation period according to Eq. 1. In contrast, the AATCC 100 rates the reduction of germs by the sample after the incubation period to the number of micro-organisms at starting point $(0 \mathrm{~h})$ as stated by Eq. 2 . While the PU-foam with TLC was able to impede $P$. aeruginosa progeny, bacteria numbers still increased over the incubation period. Hence, no antibacterial effect is found according to the AATCC 100. However, as numbers of germs in the PU-foam with TLC samples are lower than that of the polyester growth control, a significant antimicrobial activity against $P$. aeruginosa is issued according to the JIS L 1902.

That the basic materials alginate and CMC exert an antimicrobial influence at all in the challenge tests is due to their gelling properties. Alginate and CMC fibers absorb water and swell, the spaces between the fibers are closed and bacteria are trapped [20, 27, 28]. In accordance, the dressings demonstrated a significantly higher 
Fig. 4 For determination of the $\mathrm{pH}$ influence on antibacterial efficacy using the ADT, casoagar plates with different $\mathrm{pH}$ were inoculated with $S$. aureus (a) or $P$. aeruginosa (b) and incubated with the silvercontaining dressings at $37^{\circ} \mathrm{C}$ for $24 \mathrm{~h}$. Subsequently, the zone of inhibition (ZOI) was measured
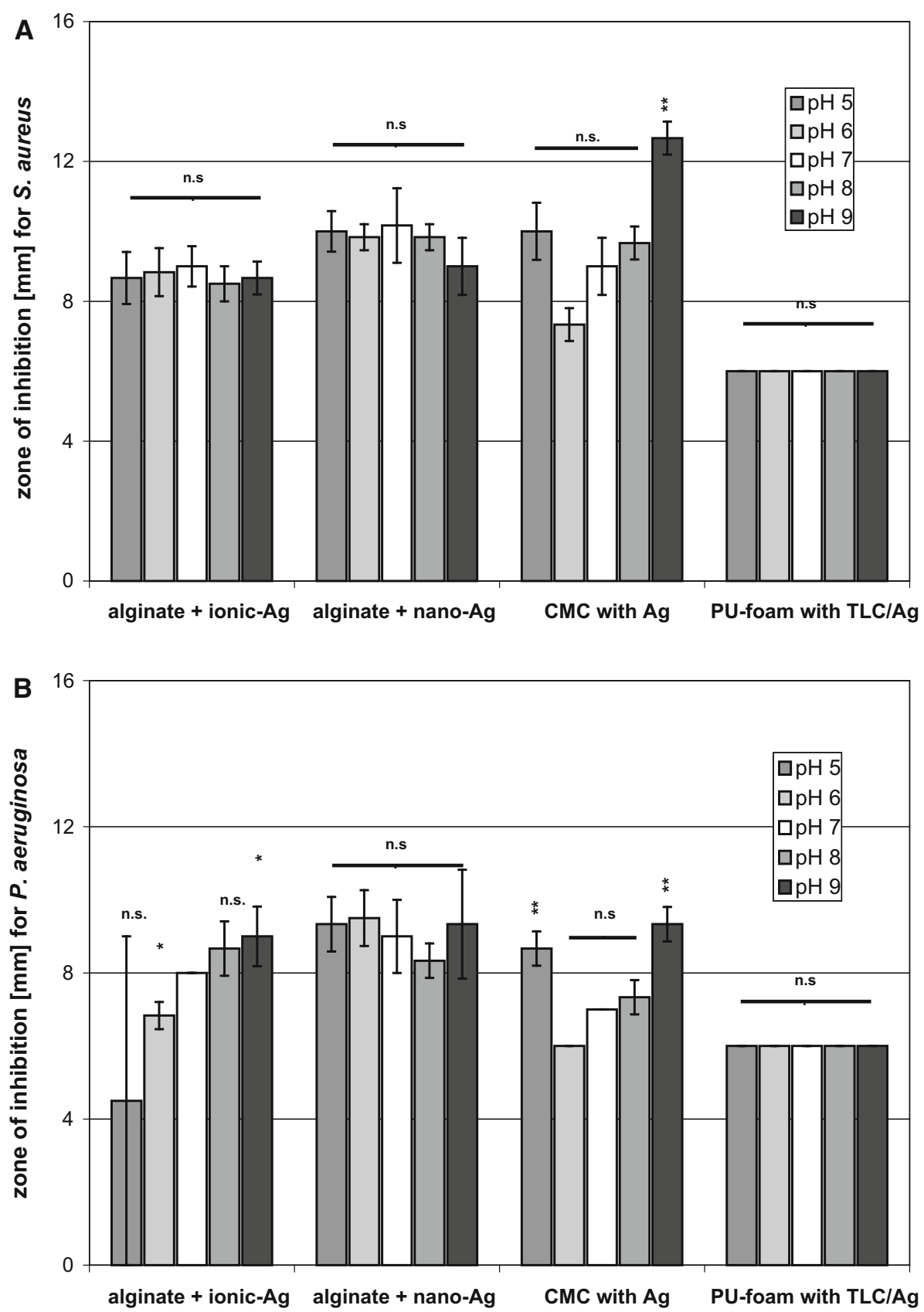

effect on $P$. aeruginosa compared to $S$. aureus. This is due to the fact that gram-negative species are far more susceptible to the water deprivation caused by the fluid uptake than the gram-positive $S$. aureus (Prof. Kramer, personal communication). Although the PU-foam with TLC does not produce a gel, it does provide a high fluid absorption capacity and might immobilize bacteria inside its matrix. This could be supported by interactions between the TLC-components of the PU-foam with the cell wall of the bacteria, which would also explain the differences observed for the gram-positive $S$. aureus and the gram-negative $P$. aeruginosa.
This study clearly demonstrates that method features have to be taken into account for selection of a specific test and interpretation of the results. As bacteria in challenge tests come into direct contact with the material, not only items containing an antimicrobial agent, such as silver, might exert an effect but also samples with high absorptive capacities that wield the ability to immobilize microorganisms. In contrast, the ADT registers only antimicrobial materials that contain a diffusible agent. It is a simple test that can be used to compare different samples under consistent conditions but does not yield a quantitative evaluation. Here, extraction-based methods such as MLN 

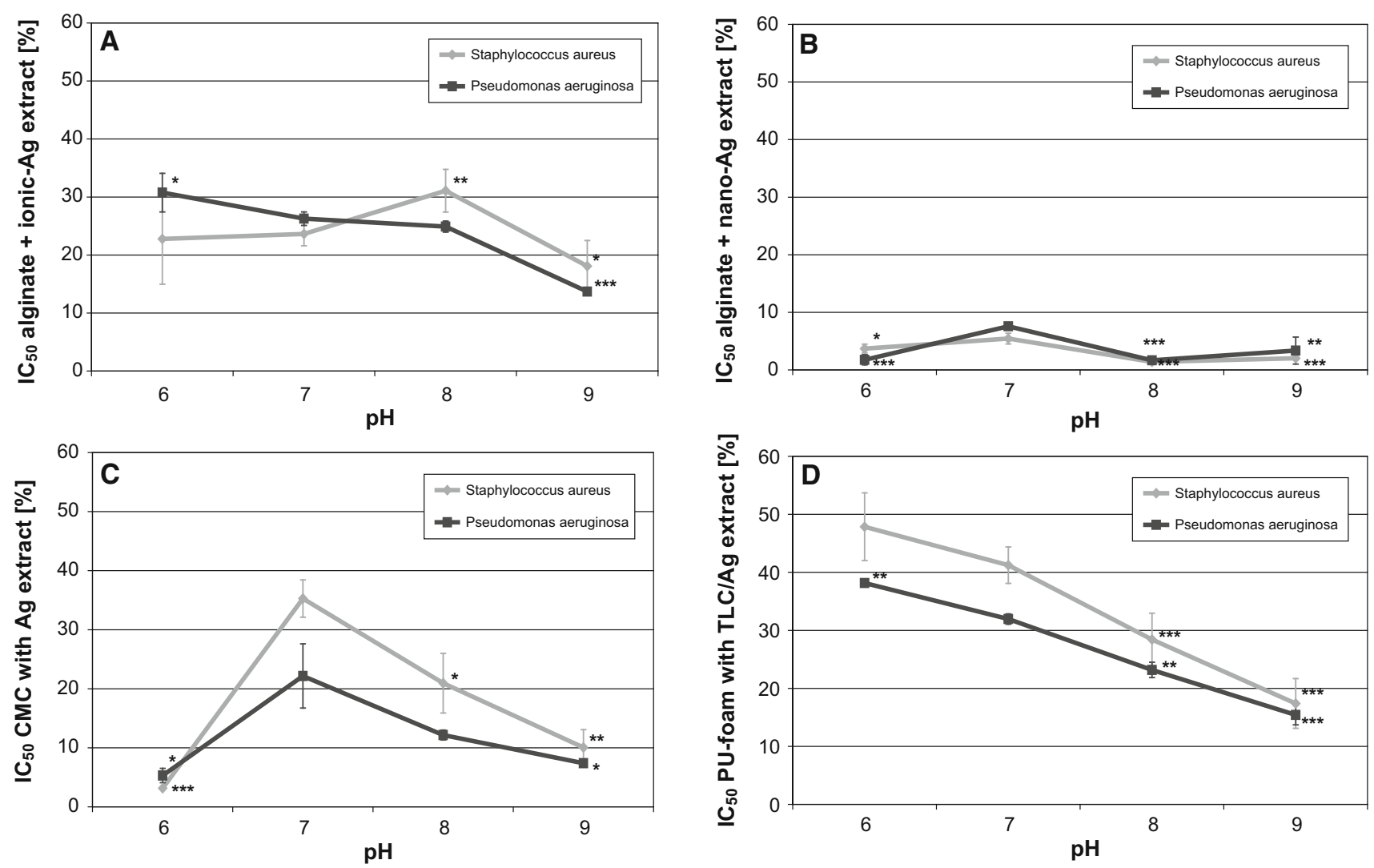

Fig. 5 The specific $\mathrm{IC}_{50}$, the half maximum inhibitory concentrations, were calculated from the dose-response curves recorded at different $\mathrm{pH}$ for the extracts of alginate + ionic-Ag (a ), alginate + nano-Ag (b), CMC with $\mathrm{Ag}^{+}$(c) and PU-foam with TLC/Ag ${ }^{+}$(d) using MLN

and LQbATP are advantageous as they allow measurement of microbial growth and quantification of viable microorganisms, respectively. However, they will also only assess a material as antimicrobially effective if it contains an extractable antimicrobial agent. To distinguish whether a material may convey an active effect or a passive mechanism, it is recommended to choose tests from both groups.

Conversely, if a specific question is raised, e.g. how the wound $\mathrm{pH}$ affects the antibacterial efficacy of antimicrobial-containing dressings, tests that address passive mechanisms are not needed while experiments that assess both, the influence on diffusion capacity and the extraction capability should be included. Hence, ADT and MLN were elected to investigate the effect of $\mathrm{pH}$ on antimicrobial activity of silver-containing dressings. In vitro studies showed that wound dressings can have significant effects on the $\mathrm{pH}[26]$. In accordance, alginate + ionic-Ag and $\mathrm{CMC}$ with $\mathrm{Ag}^{+}$led to a more acidic environment in vitro while alginate + nano- $\mathrm{Ag}$ stabilzed the $\mathrm{pH}$ around 8.0 (Table 2). PU-foam with TLC/ $\mathrm{Ag}^{+}$had no effect on the $\mathrm{pH}$. Similarly, the question has to be raised, if wound $\mathrm{pH}$ vice versa affects the activity and efficacy of antimicrobial dressings. Recent studies relating to the influence of the $\mathrm{pH}$ on the performance of antiseptics revealed that bactericidal activity of chlorhexidine and octenidine was mainly $\mathrm{pH}-$ independent in the $\mathrm{pH}$ range from 5.0 to 9.0 while a most pronounced influence was observed for polihexanide and PVP-iodine (own unpublished results). In the ADT, $\mathrm{pH}-$ independent formation of ZOI was obtained for alginate + ionic $\mathrm{Ag}$, alginate + nano- $\mathrm{Ag}$, and PU-foam with TLC/Ag ${ }^{+}$against $S$. aureus while a slight increase of the effect of CMC with $\mathrm{Ag}^{+}$was observed at $\mathrm{pH}$ 9.0. This is in accordance with a study by Braunwarth et al. which showed that silver-containing dressings possess similar bacteriostatic effects over a $\mathrm{pH}$ range of 5.5-9.0 [29]. However, against $P$. aeruginosa only alginate + nano- $\mathrm{Ag}$ and PU-foam with $\mathrm{TLC} / \mathrm{Ag}^{+}$exerted consistent effects at the different $\mathrm{pH}$ while $\mathrm{CMC}$ with $\mathrm{Ag}^{+}$demonstrated formation of larger $\mathrm{ZOI}$ at $\mathrm{pH} 5.0$ and 9.0 and alginate + ionic-Ag exhibited a significant increase of ZOI formation from $\mathrm{pH} 5.0$ to 9.0. Comparable effects were observed in the MLN measurements at different $\mathrm{pH}$ for alginate + ionic- $\mathrm{Ag}$ and $\mathrm{CMC}$ with $\mathrm{Ag}^{+}$against both, $P$. aeruginosa and $S$. aureus. $\mathrm{IC}_{50}$ values for the alginate + nano-Ag extracts were also decreased at $\mathrm{pH}$ 6.0, 8.0 and 9.0 compared to $\mathrm{pH}$ 7.0. Most notable was the effect by $\mathrm{CMC}$ with $\mathrm{Ag}^{+}$at $\mathrm{pH} 6$ which is most likely due to the 
acidification of the solution by the dressing itself (Table 2). It was found that planktonic bacteria in solution are quite sensitive to environmental changes in the $\mathrm{pH}$ [30], for instance, neither $S$. aureus nor $P$. aeruginosa could be grown in medium at $\mathrm{pH} 5.0$ for MLN measurements while no effect on these micro-organisms was found growing them on agar with $\mathrm{pH} 5.0$ for the ADT (own unpublished results). Moreover, a more alkaline environment might also increase the susceptibility of bacteria to antimicrobial agents [30]. Hence, it can be expected that dressings' effects on $\mathrm{pH}$ and release of silver ions act synergistically for antimicrobial efficacy.

\section{Conclusions}

In conclusion, it could be shown that the method selected for determination of antimicrobial effects of wound dressings will influence the results obtained and therefore, the evaluation of the antimicrobial efficacy. Hence, this has to be taken into account for selection of a specific test and the interpretation of data. The agar diffusion test ADT is a simple test that can be used to register antimicrobial materials containing diffusible agents, but it does not yield a quantitative evaluation. While challenge tests like JIS L 1902 or AATCC 100 do provide a quantitative result in form of log-reduction values or the decrease of viable germs in [\%], they also encompass the effect of materials with high absorptive capacities that wield the ability to immobilize micro-organisms. Here, the new extractionbased methods MLN (microplate-laser nephelometry) and LQbATP (luminometric quantification of bacterial ATP) can close the gap, as they only assess a material as antimicrobially effective if it contains an extractable antimicrobial agent and supply quantification of microbial growth and viable micro-organisms, respectively. In general, it can be recommended to choose several tests to distinguish whether a material may convey an active effect or a passive mechanism. Moreover, to be informative for clinical application, further tests should be performed in the presence of wound exudate and/or necrotic tissue as these may profoundly affect the antimicrobial activity in vivo.

Moreover, it is of interest to investigate the influence of the $\mathrm{pH}$ on the performance of antimicrobial wound-dressings as chronic wounds most commonly have a $\mathrm{pH}$ range of $6.5-8.5[24,25]$. This shift towards higher $\mathrm{pH}$ values in chronic wounds compared to acute wounds is called 'alkaline shift' and is thought to be due to both, tissue necrosis and the presence of microorganisms. Here, ADT and MLN were used to investigate the influence of $\mathrm{pH}$ on antimicrobial activity of selected silver-containing dressings. The results obtained show that the release of silver and hence its antimicrobial efficacy is partially $\mathrm{pH}$ - dependent. In addition, the dressings themselves may affect the $\mathrm{pH}$. From the results it can further be speculated that dressings' effects on $\mathrm{pH}$ and release of silver ions act synergistically for antimicrobial efficacy. Hence, it can be expected that the silver-containing dressings alginate + ionic- $\mathrm{Ag}$ and $\mathrm{CMC}$ with $\mathrm{Ag}^{+}$aid wound healing by antimicrobial effects of silver as well as the establishment of a low physiological $\mathrm{pH}$.

Acknowledgments This work was partially supported by Lohmann \& Rauscher GmbH \& Co. KG (Germany). The authors would like to thank Denise Reichmann for excellent technical assistance.

Open Access This article is distributed under the terms of the Creative Commons Attribution License which permits any use, distribution, and reproduction in any medium, provided the original author(s) and the source are credited.

\section{References}

1. Warriner R, Burrell R. Infection and the chronic wound: a focus on silver. Adv Skin Wound Care. 2005;18:2-12.

2. Kingsley A. The wound infection continuum and its application to clinical practice. Ostomy Wound Manage. 2003;49:1-7.

3. Wright JB, Lam K, Buret AG, Olson ME, Burrell RE. Early healing events in a porcine model of contaminated wounds: effects of nanocrystalline silver on matrix metalloproteinases, cell apoptosis, and healing. Wound Repair Regen. 2002;10:141-51.

4. Percival SL, Bowler PG, Russel D. Bacterial resistance to silver in wound care. J Hosp Infect. 2005;60:1-7.

5. Burrell RE. A scientific perspective on the use of topical silver preparations. Ostomy Wound Manage. 2003;49(5A Suppl):19-24.

6. Klančnik A, Piskernik S, Jeršek B, Smole Možina S. Evaluation of diffusion and dilution methods to determine the antibacterial activity of plant extracts. J Microbiol Method. 2010;18:121-6.

7. DIN 58940-3: Medical microbiology—susceptibility testing of microbial pathogens to antimicrobial agents-part 3: agar diffusion test.

8. DIN EN ISO 20645: Textile fabrics-determination of antibacterial activity-agar diffusion plate test.

9. AATCC 100: Assessment of antibacterial finishes on textile materials.

10. JIS L 1902: Testing for antibacterial activity and efficacy on textile products.

11. Marques SM, Esteves da Silva JC. Firefly bioluminescence: a mechanistic approach of luciferase catalyzed reactions. IUBMB Life. 2009;61:6-17.

12. Nazari M, Hosseinkhani S. Design of disulfide bridge as an alternative mechanism for color shift in firefly luciferase and development of secreted luciferase. Photochem Photobiol Sci. 2011;10:1203-15.

13. Han T, Nazarenko Y, Lioy PJ, Mainelis G. Collection efficiencies of an electrostatic sampler with superhydrophobic surface for fungal bioaerosols. Indoor Air. 2011;21:110-20.

14. Finger S, Wiegand C, Buschmann HJ, Hipler UC. Antimicrobial properties of cyclodextrin-antiseptics-complexes determined by microplate laser nephelometry and ATP bioluminescence assay. Int J Pharm. 2012;436:851-6.

15. Bevan CD, Llyod RS. A high-throughput screening method for the determination of aqueous drug solubility using laser nephelometry in microtiter plates. Anal Chem. 2000;72:1781-7. 
16. Finger S, Wiegand C, Buschmann HJ, Hipler UC. Antibacterial properties of cyclodextrin-antiseptics-complexes determined by microplate laser nephelometry and ATPbioluminescence assay. Int J Pharmaceut. 2013;452:188-93.

17. Wiegand C, Abel M, Ruth P, Hipler UC. Analysis of the adaptation capacity of Staphylococcus aureus to commonly used antiseptics by microplate laser nephelometry. Skin Pharmacol Physiol. 2012;25:288-97.

18. Seyfarth F, Schliemann S, Elsner P, Hipler UC. Antifungal effect of high- and low-molecular-weight chitosan hydrochloride, carboxymethyl chitosan, chitosan oligosaccharide and $\mathrm{N}$-acetyl-Dglucosamine against Candida albicans, candida krusei and Candida glabrata. Int J Pharm. 2008;353:139-48.

19. Fouda MMG, Knittel D, Hipler UC, Elsner P, Schollmeyer E. Antimycotic influence of $\beta$-cyclodextrin complexes-in vitro measurements using laser nephelometry in microtiter plates. Int $\mathbf{J}$ Pharm. 2006;311:113-21.

20. Wiegand C, Heinze T, Hipler UC. Comparative in vitro study on cytotoxicity, antimicrobial activity, and binding capacity for pathophysiological factors in chronic wounds of alginate and silver-containing alginate. Wound Repair Regen. 2009;17:511-21.

21. Wiegand C, Hipler UC. Evaluation of biocompatibility and cytotoxicity using keratinocyte and fibroblast cultures. Skin Pharmacol Physiol. 2009;22:74-82.

22. Kirketerp-Moller K, Jensen PO, Fazli M, Madsen KG, Pedersen J, Moser C, Tolker-Nielsen T, Hoiby N, Giuskov M, Bjarnsholt
T. Distribution, organization, and ecology of bacteria in chronic wounds. J Clin Microbiol. 2008;46:2717-22.

23. Dissemond J, Schmid EN, Esser S, Witthoff M, Goos M. Bakterielle Kolonisation chronischer Wunden. Der Hautarzt. 2004; 55:280-8.

24. Eberlein T. presented at the conference of the DGfW. 2010.

25. Dissemond J, Witthoff M, Brauns TC, Haberer D, Goos M. pHWert des Milieus chronischer Wunden. Hautarzt. 2003;54:959-65.

26. Weindorf M. pH-Werte moderner Wundauflagen und deren Einfluß auf das umgebende Milieu: Resultate einer in vitro Untersuchung. ZfW. 2007;12:92-7.

27. Qin Y. Silver-containing alginate fibres and dressings. Int Wound J. 2005;2(2):172-6.

28. Walker M, Hobot JA, Newman GR, Bowler PG. Scanning electron microscopic examination of bacterial immobilisation in a carboxymethyl cellulose (AQUACEL ${ }^{\circledR}$ ) and alginate dressings. Biomaterials. 2003;24:883-90.

29. Braunwarth $\mathrm{H}$, Brill $\mathrm{FHH}$, Brill $\mathrm{H}$. Results of in vitro testing of wound dressings with sustained release of polihexanide (PHMB) and silver-ions at different pH-values. Wund Manage. 2011;3: 119-25.

30. McDonnell G, Russell AD. Antiseptics and disinfectants: activity, action and resistance. Clin Microbiol Rev. 1999;12:147-79. 